\title{
Mechanisms of current passage and excitation of electroluminescence in GaSe:Er monocrystals
}

\author{
B.G. Tagiyev, R.S. Madatov, F.Sh. Aydayev, T.M.Abbasova \\ Radiation Researches Department of Azerbaijan Academy of Sciences, $31^{a}$ H.Javid avenue, 370143 Baku, Azerbaijan \\ Phone: (99412) 398-318; fax: (99412) 398-318; e-mail: ibrahim_gabulov@yahoo.com
}

\begin{abstract}
The paper deals with electroluminescence investigations in GaSe:Er monocrystals. It is ascertained that $\mathrm{Er}^{3+}$ centers in GaSe are excited by two ways: a) as a result of nonradiating recombination of the injected charge carrier in donor-acceptor states and, b) transfer of evolved energy by means of Coulomb interaction. The concentration of majority carriers in the current step field $\left(6.26 \cdot 10^{11} \mathrm{~cm}^{-3}\right)$ and activation energy of impurities $(0.14 \mathrm{eV})$ have been determined using data obtained.
\end{abstract}

Keywords: electroluminescence, monocrystal, intra-central transitions, luminescent centers, excitation

Paper received 14.06.02; accepted for publication 10.12.02.

\section{Experimental results}

The band gap for most of $\mathrm{A}^{\mathrm{III}} \mathrm{B}^{\mathrm{VI}}$ semiconductors allows to excite many metastable states of rare earth elements. It enables to investigate the luminescent properties of rare earths in these semiconductors.

Photoluminescence and electroluminescence $L_{R}^{3+}$ in $\mathrm{GaS}$ and $\mathrm{GaSe}$ monocrystals have been investigated in the works [1-4]. It is shown that rare earth luminescent centers are excited due to pumping into the conduction band through local states in the band gap of a matrix, and through both impurity and direct excitation of luminescent centers, as well. It is prompted us to investigate the electroluminescence of intra-central transitions $E_{R}^{3+}$ in GaSe:Er monocrystals, as the creation of electroluminescent materials operating in the various spectral ranges is of a large practical interest.

Used single crystals were grown by the Bridgman method. Impurities in the form of metal were introduced into the working mass before the synthesis. Contacts to the samples were created by fusion and sputtering of indium on opposite mirror surfaces, which were perpendicular to the axis $\overrightarrow{\mathrm{C}}$ of the monocrystal. Impurity concentration were determined by chemical-spectral analysis and it varied within $\left(8 \cdot 10^{17} \div 5.4 \cdot 10^{18} \mathrm{~cm}^{-3}\right)$.

Electroluminescence of intra-central transitions $E_{R}^{3+}$ in GaSe:Er single crystals and electrical properties of these crystals were investigated in the present work.

The electroluminescence spectrum for GaSe:Er monocrystals is shown in Fig. 1. The samples with contacts from indium in the sandwich construction are immersed into the liquid nitrogen and then they are excited by both pulsed and constant electric fields. Electroluminescence was registered using photomultipliers EMP-100, EMP-61 and a photoresistor $\mathrm{PbS}$.

As it is seen from Fig. 1, pronounced intra-central transitions ${ }^{4} I_{9 / 2}{ }^{4} I_{11 / 2}{ }^{4} I_{13 / 2} \rightarrow{ }^{4} I_{15 / 2} E_{R}^{3+}$ are observed. Electroluminescence intensity always depends super-linearly $(n=1.4)$ on the current passing through the sample.

Electrical properties of In-GaSe:Er-In structures were studied with the purpose to clarify the excitation mechanism of electroluminescence.

Volt-ampere characteristics for undoped (curve 1) and doped by erbium GaSe single crystals (curves 2,3) are depicted in Fig. 2a. Current regime was limited by a space charge region inherent to undoped samples (discrete level $E_{t}=0.69 \mathrm{eV}$; concentration $N_{t}=5.7 \cdot 10^{13} \mathrm{~cm}^{-3}$ ). The corresponding capture factor calculated from quadratic trap realm was equal to $2.2 \cdot 10^{-6}$.

Strongly expressed characteristic parts of the current that is limited by space charge are not detected (curves 2 and 3 ) in the case of doped samples. Moreover, both curves are situated over the non-trap part of the current. This part of the current was calculated for GaSe:Er sample $\left(5.4 \cdot 10^{18} \mathrm{~cm}^{-3}, L=90\right.$ micrometers, dotted curve). The capture factor for doped samples increases with increasing temperature and at the value $386 \mathrm{~K}$ is equal to $1.6 \cdot 10^{4}$. The value of the capture factor is considerably more than unity at low temperatures ( $T=102 \mathrm{~K})$.

Volt-ampere characteristics were determined in the wide temperature range (between 100 to $390 \mathrm{~K}$ ). Temperature dependences of electroconductivities for different samples (Fig. 2b) were determined from ohmic parts of volt-ampere characteristics under various temperatures. 


\section{B.G. Tagiyev et al.: The mechanisms of current passage and excitation...}

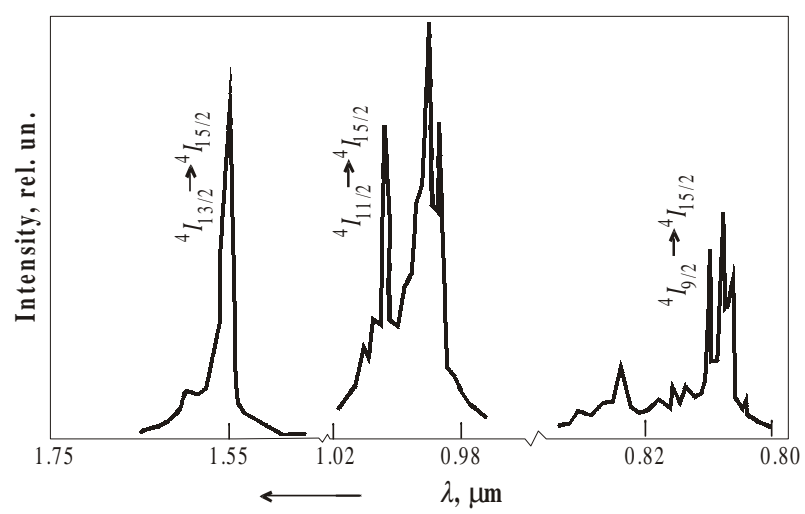

Fig. 1. Electroluminescence spectra of GaSe:Er monocrystals at $T=77 \mathrm{~K}$

Energy levels in doped GaSe monocrystals were determined from the slope of the electroconductivity dependence and they lie in the interval from 0.05 to $0.70 \mathrm{eV}$. Any energy levels near the value $0.28 \mathrm{eV}$ were not detected in GaSe monocrystals doped by In.

As $L_{R}^{3+}$ is an isovalent impurity for GaSe [1-4], such jump increase of electroconductivity (by $4-5$ order) (Fig. $2 \mathrm{~b}$ ) and decrease of activation energy up to $0.28 \mathrm{eV}$ are explained by removal of donor background due to chemical activity of $L_{n}^{3+}$ to the uncontrolled impurities and also moving off the Fermi level from the bottom of the conduction band.
The fact of kindling exciton glow line when doping the crystals by $L_{n}$ is a confirmation of donor background removal [1].

The dependence $j(u)$ were constructed in coordinates connected with the level ionization process by strong electric field. This dependence is nonlinear.

Moreover, medium fields in the sample $\left(E_{\max }=2 \cdot 10^{2} \mathrm{~V} / \mathrm{cm}\right)$ are much less than ionization fields $\left(E=10^{4} \mathrm{~V} / \mathrm{cm}\right)$.

The methods developed in [5] were used for an analysis of sample volt-ampere characteristics. Determination of the dependence of the volt-ampere characteristic exponent $(\alpha)$ on voltage (Fig. 2a, curve 4 ) is put into a basis of the analysis in these methods. Discrimination of the electroconductivity coefficient was determined. It is equal to $Q_{m}=1.1 \cdot 10^{-3}$. Realization of $Q_{m}<<1$ condition and observation of electroluminescence under low electrical fields showed that current transport in In-GaSe:Er-In structures is stipulated by double injection [5]. Injected carriers of both types are captured to all possible traps, and these carriers partly recombine. Further, excitation is transmitted to the activator centers by the following ways: 1) by means of Coulomb interaction transferring $E_{R}^{3+}$ from the basic state into the excited one; 2) by charge carrier in the nearest donor or acceptor, which is ejected into the appropriate semiconductor band; 3) using radiative recombination of injected carriers by donoracceptor couples. Non-radiative energy transfer was de-
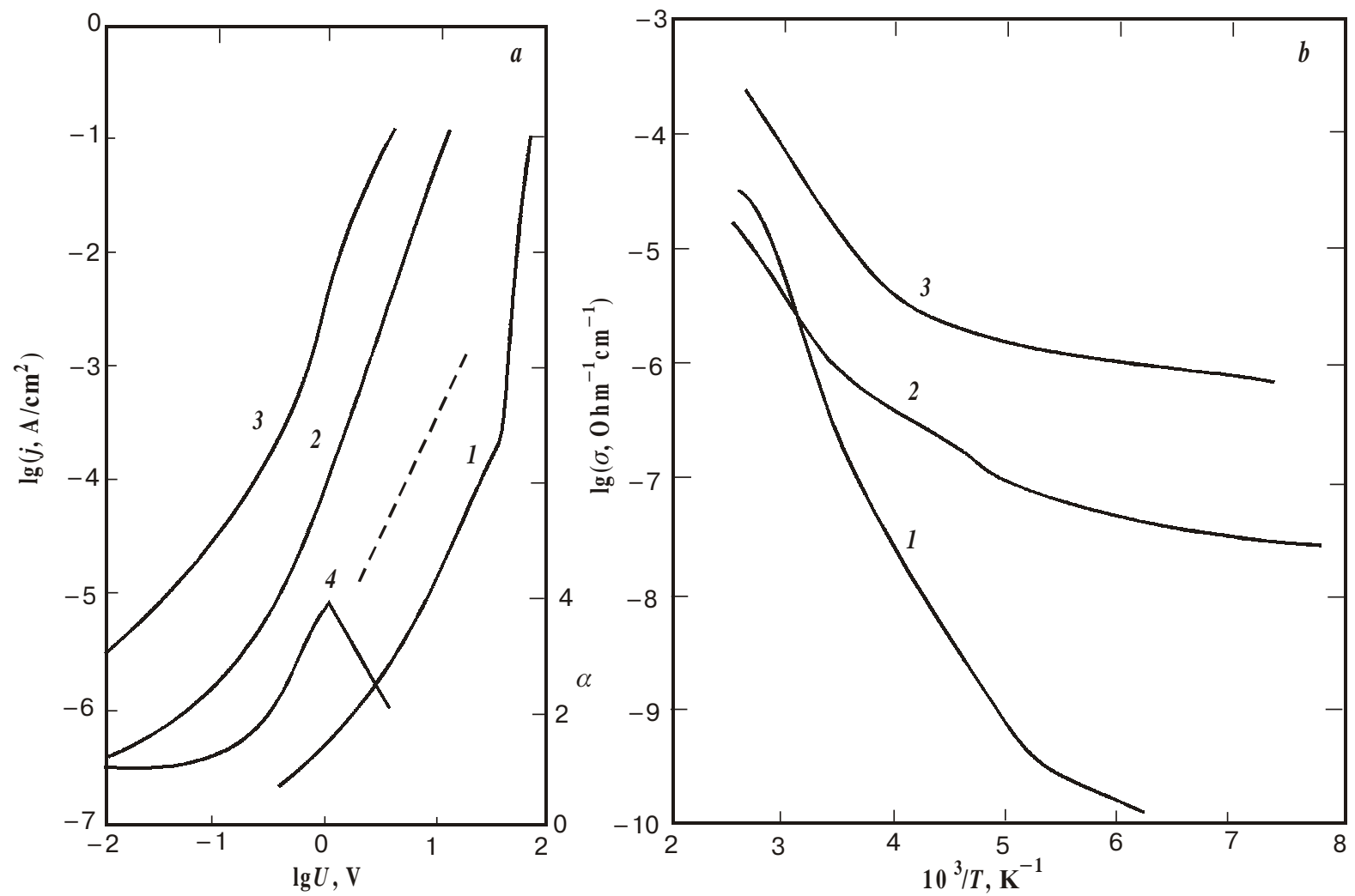

Fig. 2. $a-1,2,3$ - volt-ampere characteristics of GaSe monocrystals undoped $(I)$ and doped $(2,3)$ by erbium $\left(1.2 \cdot 10^{18}\right.$ and $5.4 \cdot 10^{18}$ $\mathrm{cm}^{-3}$, respectively); 4 - dependence of volt-ampere characteristic exponent on voltage; dotted line - non-trap part of the current calculated for GaSe:Er sample $\left(5.4 \cdot 10^{18} \mathrm{~cm}^{-3}\right) ; b$ - temperature dependence of electroconductivity for GaSe monocrystals undoped (1) and doped by erbium $(2,3)$. 


\section{B.G. Tagiyev et al.: The mechanisms of current passage and excitation...}

termined by a character of luminescence intensity decrease of the wide-band matrix while doping it by $E_{R}^{3+}$. Such energy transfer excludes second version of an energy transfer to $E_{R}^{3+}$ in $G a S e$.

The majority carrier concentration in the area of current step $\left(6.26 \cdot 10^{11} \mathrm{~cm}^{-3}\right)$, trap depth $(0.14 \mathrm{eV})$, the value of $\mu_{n} \tau_{n m}$ for majority carriers $\left(6.1 \cdot 10^{-5} \mathrm{~cm} \mathrm{~V}^{-1}\right)$ was determined by known parameters of the current step $U_{m}, j_{m}$, $\alpha_{m}$, and some Stark's components of terms, such as ${ }^{4} I_{9 / 2}$ $\left(12305,12395,12445 \mathrm{~cm}^{-1}\right),{ }^{4} I_{11 / 2}(10200,10135,10185$ $\left.\mathrm{cm}^{-1}\right),{ }^{4} I_{13 / 2}\left(6580,6655,6740 \mathrm{~cm}^{-1}\right)$ and ${ }^{4} I_{15 / 2}(0,95$, $145,185,220,285,340,400 \mathrm{~cm}^{-1}$ ) $\mathrm{Er}^{3+}$ determined by spectra of excitation and electroluminescence.

\section{Conclusions}

Thus, it is ascertained that $\mathrm{Er}^{3+}$ centers in GaSe are excited as a result of both non-radiative recombination of injected charge carriers by donor-acceptor states and transfer of evolved energy by means of Coulomb interaction.

\section{References}

1. T.B.Tagiyev, F.Sh. Aydayev, Electroluminescence and injection current in GaS:Ko/l Physics and Technique of Semiconductors, 20 (4), pp. 723-726 (1986).

2. T.B.Tagiyev, G.M. Niftiyev, F.Sh. Aydayev, Photo- and electroluminescence of $\mathrm{Er}^{3+}$ in $\mathrm{GaS}$ and GaSe monocrystals// Physics and Technique of Semiconductors, 20 (4), pp. 780 (1986).

3. T.B.Tagiyev, G.M. Niftiyev, F.Sh. Aydayev. V.F. Zolin, Ch.M Briskina, V.M. Markushev, Photo- and electroluminescence of $\mathrm{Er}^{3+}$ in GaS monocrystals // Opt. and spectr. 62(2), pp. 461463 (1987).

4. T.B.Tagiyev, F.Sh. Aydayev, Quantum yield and cross-section of shock-excitation by rare earths in gallium sulfide monocrystals // Applied spectroscopy 52(2), pp. 314-316 (1990).

5. A.I. Zuganov, S.V. Svechnikov, Injection-contact phenomena in semiconductors, Kyiv, Naukova dumka, 1981, 282p. 